\title{
Stability of localized modes in rotating tokamak plasmas
}

\author{
J W Haverkort ${ }^{1,2}$ and $\mathbf{H}$ J de Blank ${ }^{1}$ \\ ${ }^{1}$ FOM Institute for Plasma Physics Rijnhuizen, Association EURATOM-FOM, Trilateral \\ Euregio Cluster, PO Box 1207, Nieuwegein, The Netherlands \\ ${ }^{2}$ Centrum Wiskunde \& Informatica (CWI), PO Box 94079, Amsterdam, The Netherlands \\ E-mail: j.w.haverkort@cwi.nl
}

Received 15 October 2010, in final form 14 December 2010

Published 14 March 2011

Online at stacks.iop.org/PPCF/53/045008

\begin{abstract}
The ideal magnetohydrodynamic stability is investigated of localized interchange modes in a large-aspect ratio tokamak plasma. The resulting stability criterion includes the effects of toroidal rotation and rotation shear and contains various well-known limiting cases. The analysis allows for a general adiabatic index, resulting in a stabilizing contribution from the convective effect. A further stabilizing effect from rotation exists when the angular frequency squared decreases radially more rapidly than the density. Flow shear, however, also decreases the stabilizing effect of magnetic shear through the KelvinHelmholtz mechanism. Numerical simulations reveal the merits and limitations of the performed local analysis.
\end{abstract}

\section{Introduction}

Current tokamaks often show significant toroidal flow and flow shear, which can have a large impact on stability. Rotation was found to stabilize the resistive wall mode, see, e.g. [1] and references therein. Flow shear is well known for its role in turbulence suppression, leading to transport barriers. A mitigating influence of flow shear has also been observed for instabilities, such as tearing modes [2].

A variety of essentially hydrodynamic instabilities has been investigated experimentally in magnetically confined plasmas. The rotational or centrifugal Rayleigh-Taylor instability e.g. almost always appears in field-reversed configurations [3] and has recently been observed in a magnetic dipole experiment [4]. This instability, together with the Kelvin-Helmholtz instability [5] has been extensively studied in the 1970s and 1980s in $Q$-machines, see, e.g. $[6,7]$. More recently, the Kelvin-Helmholtz instability associated with flow shear has been considered to play a role in the scrape-off layer of tokamak plasmas [8-10].

Finally, when rotation approaches the ion sound velocity, the centrifugal convective effect can play a stabilizing role. This is sometimes referred to as gyroscopic stabilization after an 
analogy from the original paper of [11]. Observations consistent with this predicted effect are reported for sawteeth in MAST [12,13], TEXTOR [14], and recently for certain ideal magnetohydrodynamic (MHD) modes [15].

Pressure-driven instabilities put an upper limit on the maximum attainable dimensionless pressure $\beta$, thereby limiting the performance of a tokamak as an economically viable fusion energy source. In a magnetically confined toroidal plasma, various magnetohydrodynamic instabilities can only develop around rational surfaces. At these magnetic surfaces, the main poloidal and toroidal Fourier harmonics $m$ and $n$ of the unstable mode match the local pitch $q \approx m / n$ of the magnetic field, so that magnetic field line bending is minimal.

In this paper we investigate the influence of toroidal rotation on pressure-driven instabilities localized near rational surfaces. We use a large-aspect ratio expansion of the linear MHD equations to derive an insightful stability criterion. Numerical simulations serve to test and illustrate selected aspects of the analysis. Both the analytical and the numerical investigations include flow self-consistently and take into account plasma compressibility.

For high $n$, coupling between poloidal harmonics will enable a mode to balloon outwards into the low-field region of unfavourable curvature. Ballooning modes were found to be further destabilized by rigid rotation $[16,17]$, primarily by a further enhancement of the unfavourable curvature. Flow shear was often found to have a stabilizing influence [18-20]. Unless the magnetic shear is very low, this effect may, however, be small for sub-Alfvénic flow shear [18]. In specific cases the net effect of flow shear was found to be destabilizing [21].

It was found that, at low [22] or reversed [23] magnetic shear, over a large range of parameters the most unstable modes are low or intermediate $n$ 'infernal modes' that can be unstable well below the ballooning mode stability limit. These ideal [24] or resistive [25] modes are driven by pressure gradients and are capable of causing serious disruptions and collapses. For $m=n=1$ this mode is called the quasi-interchange mode [26,27], which was used to create an understanding of sawtooth oscillations.

Often a local analysis is performed in which unstable modes are assumed to be highly localized around a rational magnetic surface. An analysis of this kind lead to the Mercier criterion [28]. For a tokamak plasma with a circular cross-section, a large-aspect ratio $\epsilon^{-1}$, and low $\beta \sim \epsilon^{2}$, a simple stability criterion results $[29,30]$ that relates the maximum allowed pressure gradient to the local magnetic shear. The only difference between this toroidal result and the cylindrical Suydam criterion [31-33] is a stabilizing term due to the average curvature of the toroidal magnetic field. Corrections to the cylindrical result due to plasma flow were obtained in $[34,35]$. Corrections to the toroidal result due to flow shear [36] or centrifugal forces $[37,38]$ have only been obtained separately and for a ratio of specific heats $\gamma=1$.

We will generalize these results to include the effects of both flow shear and centrifugal forces. Furthermore, the present analysis allows for an adiabatic index larger than $\gamma=1$. The most notable effect associated with a deviation from isothermality is the convective effect considered experimentally in [12-15]. This effect was in analytical and numerical studies found to stabilize the internal kink mode [11,39], localized modes [38], and the quasi-interchange mode [40]. We find that, depending on the specific profiles, the net effect of toroidal rotation may be either stabilizing or destabilizing. The obtained stability criterion will be compared with and supplemented with numerical simulations.

\section{Preliminaries}

We consider the ideal magnetohydrodynamic equilibrium of an axisymmetric tokamak plasma with major radius $R_{0}$ and minor radius $a$. We use a cylindrical coordinate $\operatorname{system}(R, Z, \phi)$ and consider plasma rotation with an angular frequency $\Omega$ in the toroidal $\phi$-direction. Because 
of the high thermal conductivity along the magnetic field, we can assume the equilibrium temperature $T=p / \rho$ to be constant within magnetic surfaces labelled by $\psi$. In order to satisfy the force balance $\partial p /\left.\partial R\right|_{\psi}=\rho R \Omega^{2}$, the pressure $p$ and density $\rho$ satisfy

$$
p / p_{\mathrm{s}}=\rho / \rho_{\mathrm{s}}=\exp \left(\mathcal{M}^{2}\left(R^{2}-R_{0}^{2}\right) / R_{0}^{2}\right),
$$

where $p_{\mathrm{s}}$ and $\rho_{\mathrm{s}}$ correspond to the static situation $\mathcal{M}=0$. Here $\mathcal{M} \equiv R_{0} \Omega / \sqrt{2 T}$ is related to the Mach number $M=R \Omega / \sqrt{\gamma T}$ by $\mathcal{M}=\sqrt{\gamma / 2} M_{0}$, where $M_{0} \equiv M\left(R_{0}\right)$. We note that $p_{\mathrm{s}}, \rho_{\mathrm{s}}, \Omega$ and $T$ are functions of $\psi$ only so that $\mathcal{M}=\mathcal{M}(\psi)$ but $M=M(R, \psi)$.

To investigate the waves and instabilities that arise in a given equilibrium, we consider an infinitesimal plasma perturbation

$$
\boldsymbol{\xi}(\psi, \theta, \phi, t)=\sum_{m} \boldsymbol{\xi}_{m}(\psi) \mathrm{e}^{\mathrm{i}(m \theta-n \phi-\omega t)},
$$

where $\theta$ is the poloidal angle in the $(r, \theta)$ flux coordinate system introduced in [41]. The linearized equation of motion for $\boldsymbol{\xi}$ was given by Frieman and Rotenberg and can for a purely toroidal rotation velocity $\boldsymbol{v}=\boldsymbol{\Omega} \times \boldsymbol{R}$ be written as [11,42]

$$
-\rho \omega_{\mathrm{D}}^{2} \boldsymbol{\xi}-2 \mathrm{i} \rho \omega_{\mathrm{D}} \boldsymbol{\Omega} \times \boldsymbol{\xi}=\boldsymbol{F}_{\mathrm{s}}(\boldsymbol{\xi})-\boldsymbol{R} \nabla \cdot\left(\rho \Omega^{2} \boldsymbol{\xi}\right) .
$$

Here $\omega_{\mathrm{D}} \equiv \omega+n \Omega$ is the Doppler-shifted frequency, the second term represents the Coriolis effect, and the right-hand side contains the static force operator $\boldsymbol{F}_{\mathrm{s}}$ and an additional force due to rotation.

The equation of motion (3) can be simplified for a tokamak plasma with a circular crosssection and inverse aspect ratio $\epsilon \equiv a / R_{0} \ll 1$, when investigating low frequencies $\omega \sim \Omega$ and assuming the ordering $\mathcal{M} \sim 1, m / q-n \sim \epsilon$ and $\beta \sim \epsilon^{2}$. Here $\beta(r)=2 \mu_{0} p_{\mathrm{s}} / B_{0}^{2}$ and $q(r)=\mathrm{d} \phi / \mathrm{d} \theta \approx r B_{\phi} / R_{0} B_{\theta}$, where the radial coordinate $r$ replaces $\psi$ and the constant $B_{0}$ refers to the magnetic field at the geometric axis $R=R_{0}$. Expanding (3) up to fourth order in $\epsilon$, for non-zero $m$ and $n$ a mode equation can be obtained for the radial component of the main harmonic $\xi \equiv \xi_{m}^{r}(r)[41]$ :

$$
\left(r^{3} A_{1} \xi^{\prime}\right)^{\prime}+\xi\left[r^{2} A_{2}^{\prime}-r\left(m^{2}-1\right) A_{1}\right]=g .
$$

Here a prime denotes $\mathrm{d} / \mathrm{d} r$ and

$$
\begin{aligned}
& A_{1}=\frac{\left(\omega_{\mathrm{D}}^{2}-\omega_{-}^{2}\right)\left(\omega_{\mathrm{D}}^{2}-\omega_{+}^{2}\right)}{\omega_{\mathrm{A}}^{2}\left(\omega_{0}^{2}-\omega_{\mathrm{D}}^{2}\right)}, \\
& A_{2}=\left.A_{1}\right|_{q=m / n}+q^{2} A-n^{2} \beta\left(1-q^{2}\right)-m^{2} \beta_{k},
\end{aligned}
$$

where

$$
\begin{aligned}
& A=\frac{\omega_{0}^{2}}{\omega_{\mathrm{A}}^{2}} \frac{4 n \Omega \omega_{\mathrm{D}}}{\omega_{0}^{2}-\omega_{\mathrm{D}}^{2}}\left(1+\frac{\Omega^{2}}{2 \omega_{\mathrm{s}}^{2}}\right), \\
& \beta_{k}=\beta \mathcal{M}^{2}\left(1+\mathcal{M}^{2}\right) .
\end{aligned}
$$

Here $\omega_{\mathrm{A}}(r) \equiv B_{0} / \sqrt{\rho_{\mathrm{s}}} R_{0}, \omega_{\mathrm{s}}(r) \equiv \sqrt{\gamma T} / R_{0}$, and

$$
\omega_{ \pm}^{2}=\omega_{\mathrm{s}}^{2}\left(a_{1} \pm \sqrt{a_{1}^{2}-a_{2}}\right) \quad \text { and } \quad \omega_{0}^{2}=\omega_{\mathrm{s}}^{2} / q^{2} \text {, }
$$

where

$$
\begin{aligned}
& a_{1}=1+\frac{1}{2 q^{2}}+\frac{1}{\gamma}\left(4 \mathcal{M}^{2}+\mathcal{M}^{4}\right)+\frac{\omega_{\mathrm{A}}^{2}}{2 \omega_{\mathrm{s}}^{2}}\left(\frac{m}{q}-n\right)^{2}, \\
& a_{2}=\frac{2(\gamma-1) \mathcal{M}^{4}}{\gamma^{2} q^{2}}+\frac{\omega_{\mathrm{A}}^{2}}{q^{2} \omega_{\mathrm{s}}^{2}}\left(\frac{m}{q}-n\right)^{2} .
\end{aligned}
$$


The notation $\left.A_{1}\right|_{q=m / n}$ means that in the evaluation of $A_{1}, q$ has to be replaced by $m / n$. The inhomogeneous term $g$ arises from the toroidal coupling of $\xi$ to its sideband amplitude $\xi_{m+1}^{r}$ [41]. When the sideband rational surface at $q=(m+1) / n$ is not located within the plasma, this coupling term is given by

$$
g=\frac{1}{2}(1+m) n^{2} q^{4} R_{0}^{2}\left(\frac{r}{a}\right)^{m+1} \tilde{\beta}^{\prime} \int_{0}^{a}\left(\frac{r}{a}\right)^{m+1} \tilde{\beta}^{\prime} \xi \mathrm{d} r
$$

where $\tilde{\beta} \equiv \beta\left(1+\mathcal{M}^{2}\right)$. We note that (4) can alternatively be written in terms of $\chi \equiv r \xi$ as

$$
r\left(r A_{1} \chi^{\prime}\right)^{\prime}+\chi\left(r\left(A_{2}-A_{1}\right)^{\prime}-m^{2} A_{1}\right)=g .
$$

Because of the last terms in $a_{1}$ and $a_{2}$ it holds that $A_{1}=\left.A_{1}\right|_{q=m / n}+(m / q-n)^{2}$. Within the present ordering $(m / q-n)^{2}=n^{2}(\Delta q)^{2} / q^{2}$, with $\Delta q$ being the difference between $q$ and its rational surface value $m / n$. Apart from $\Delta q$ in this magnetic shear term, in all other expressions $q$ refers to the constant value $q=m / n$.

Note that no assumption is made on the magnitude of the magnetic shear. It is only required that $m / q-n \sim \epsilon$ in the region where the mode amplitude is significant. Such modes experience little magnetic field line bending so that they can easily be unstable to interchanging adjacent plasma elements. Such interchange modes are sometimes referred to as flute instabilities. In the following section we will derive a stability criterion for such modes.

\section{Derivation of a stability criterion}

Under certain conditions, the continuum of frequencies $\omega_{\mathrm{D}}^{2}=\omega_{-}^{2}(r)$ will contain an accumulation point for instabilities. Equation (9) shows that for $\gamma>1, \omega_{-}^{2}$ is lifted by rotation to a finite buoyancy frequency, or Brunt-Väisälä frequency, associated with a stable entropy stratification [41,43]. A stabilizing influence can be therefore be expected for instabilities clustering at this continuum. We will consider the stability of modes that are radially highly localized, without taking into account the coupling between poloidal harmonics leading to poloidal localization. For such localized modes, the Coriolis shift [44] is much smaller than the Doppler shift so that we can assume that marginal stability holds for $\omega_{\mathrm{D}} \approx 0$. When $\gamma>1$, the $\omega_{-}$-continuum will not vanish at the rational surface. Modes clustering at this continuum are therefore not automatically unstable.

\subsection{A local mode equation}

We investigate the mode equation (13) around the radial position $r=r_{0}$ where the coefficient $r^{3} A_{1}$, multiplying the highest derivative of $\xi$, has a local minimum. Marginally stable modes will have a Doppler-shifted frequency $\omega_{\mathrm{D}} \approx n \Omega^{\prime} x$ that vanishes at $x \equiv r-r_{0}=0$. To second order in $x$, (5) gives

$$
\begin{aligned}
A_{1} & =\frac{\omega_{-}^{2} \omega_{+}^{2}}{\omega_{\mathrm{A}}^{2} \omega_{0}^{2}}\left(1+\frac{\left(n \Omega^{\prime}\right)^{2}}{\omega_{0}^{2}} x^{2}\right)-\frac{\omega_{-}^{2}+\omega_{+}^{2}}{\omega_{\mathrm{A}}^{2} \omega_{0}^{2}}\left(n \Omega^{\prime}\right)^{2} x^{2} \\
& =\frac{q^{2} a_{2} \omega_{\mathrm{s}}^{2}}{\omega_{\mathrm{A}}^{2}}\left(1+\frac{\left(n \Omega^{\prime}\right)^{2}}{\omega_{0}^{2}} x^{2}\right)-\frac{2 q^{2} a_{1}}{\omega_{\mathrm{A}}^{2}}\left(n \Omega^{\prime}\right)^{2} x^{2} \\
& =G+\left(\frac{m}{q}-n\right)^{2}+\left(\frac{G}{\omega_{0}^{2}}-\frac{F}{\omega_{\mathrm{A}}^{2}}\right)\left(n \Omega^{\prime}\right)^{2} x^{2}
\end{aligned}
$$

where

$$
\begin{aligned}
& \left.F \equiv 2 q^{2} a_{1}\right|_{q=m / n}=1+2 q^{2}\left[1+\gamma^{-1} \mathcal{M}^{2}\left(4+\mathcal{M}^{2}\right)\right] \\
& \left.G \equiv\left(\omega_{\mathrm{s}}^{2} / \omega_{\mathrm{A}}^{2}\right) q^{2} a_{2}\right|_{q=m / n}=\left(1-\gamma^{-1}\right) \beta \mathcal{M}^{4}
\end{aligned}
$$


$F$ is a toroidal inertial enhancement factor that reduces to the Pfirsch-Schlüter factor $1+2 q^{2}$ for $\mathcal{M}=0$. $G$ is the value of $A_{1}$ at the rational surface for $\omega_{\mathrm{D}}=0$. For $\gamma>1$, there will in general be a small difference $\Delta r \equiv r_{0}-r_{\mathrm{s}}$ between the rational surface at $r=r_{\mathrm{s}}$ and $x=0$ so that $(m / q-n)^{2}=\left(n q^{\prime} / q\right)^{2}\left(x^{2}+2 x \Delta r+(\Delta r)^{2}\right)$. Using (16), we can write to second order in $x$

$$
r^{3} A_{1} \approx r_{0}^{3}\left(1-\tilde{M}_{\mathrm{A}}^{2}\right)\left(\frac{n q^{\prime}}{q}\right)^{2}\left(\delta^{2}+x^{2}\right)
$$

where, evaluated at $x=0$,

$$
\begin{aligned}
& \tilde{M}_{\mathrm{A}}^{2} \equiv M_{\mathrm{A}}^{2}-M_{\mathrm{B}}^{2} \equiv\left(\frac{q}{q^{\prime}}\right)^{2} \Omega^{\prime 2}\left(\frac{F}{\omega_{\mathrm{A}}^{2}}-\frac{G}{\omega_{0}^{2}}\right) \\
& \delta^{2}=\frac{G}{1-\tilde{M}_{\mathrm{A}}^{2}}\left(\frac{q}{n q^{\prime}}\right)^{2} .
\end{aligned}
$$

In writing (19), we assumed that

$$
\frac{(\Delta r)^{2}}{\left|1-\tilde{M}_{\mathrm{A}}^{2}\right|} \ll \delta^{2} \ll r_{0}^{2} .
$$

The first inequality was used to neglect the magnetic field line bending at $x=0$ to write $A_{1}(x=0)=G$. The second inequality was used to neglect the contribution to (19) due to the variation in $r^{3}$. Finally, we assumed the contribution of $G^{\prime \prime}$ to be negligible. The implications of the orderings of (22) will be discussed in sections 3.2 and 3.4, respectively. Using $\beta / \omega_{0}^{2}=2 q^{2} / \gamma \omega_{\mathrm{A}}^{2}$ we can write

$$
\begin{aligned}
& \tilde{M}_{\mathrm{A}}^{2} \equiv \tilde{F}\left(\frac{q}{q^{\prime}}\right)^{2} \frac{\Omega^{\prime 2}}{\omega_{\mathrm{A}}^{2}}, \\
& \tilde{F}=1+2 q^{2}\left[1+\gamma^{-1} \mathcal{M}^{2}\left(4+\gamma^{-1} \mathcal{M}^{2}\right)\right] .
\end{aligned}
$$

Note the small difference in this modified inertial enhancement factor $\tilde{F}$ compared with $F$ from (17). Using (19), the mode equation (4) can locally be written as

$$
\left(\left(\delta^{2}+x^{2}\right) \xi^{\prime}\right)^{\prime}+D \xi=h
$$

where $h \equiv g\left(q / n q^{\prime}\right)^{2} /\left(1-\tilde{M}_{\mathrm{A}}^{2}\right)$ and, evaluated at $x=0$,

$$
D=\frac{r A_{2}^{\prime}-\left(m^{2}-1\right) G}{1-\tilde{M}_{\mathrm{A}}^{2}} \frac{1}{r^{2}}\left(\frac{q}{n q^{\prime}}\right)^{2} .
$$

With $\omega_{\mathrm{D}}=n \Omega^{\prime} x$, the quantity $A^{\prime}$ at $x=0$ is given by

$$
A^{\prime}=n^{2} \frac{4 \Omega \Omega^{\prime}}{\omega_{\mathrm{A}}^{2}}\left(1+\frac{\Omega^{2}}{2 \omega_{\mathrm{s}}^{2}}\right) .
$$

We note that instead of (4) the mode equation (13) for $\chi=r \xi$ could have been used. Where $\left(r A_{1}\right)^{\prime}=0$, we can write $r\left(A_{2}-A_{1}\right)^{\prime}-m^{2} A_{1}=r A_{2}^{\prime}-\left(m^{2}-1\right) A_{1}$, yielding again (25) and (26).

\subsection{Localized mode solutions}

The solutions to the local equation (25) are

$$
\xi(x)=c_{1} P_{\nu}\left(\frac{\mathrm{i} x}{\delta}\right)+c_{2} Q_{\nu}\left(\frac{\mathrm{i} x}{\delta}\right)+\frac{h}{D},
$$


where the degree of the Legendre functions $P$ and $Q$ is $v=-1 / 2+\sqrt{1 / 4-D}$. When $\gamma=1$ or $\mathcal{M}=0, \delta$ vanishes and these solutions become $x^{\nu_{ \pm}}$with $v_{ \pm}=-1 / 2 \pm \sqrt{1 / 4-D}$. For $D>1 / 4$ these solutions are rapidly oscillating and diverging near $x=0$. The solution (28) has a characteristic length scale $\delta$. In line with the local character of the present analysis, (22) requires that $\delta \ll r_{0}$. From (21) we see that magnetic shear ensures mode localization, especially for high $n$. Also, the closer the mode frequency is to that of the continuum, as measured by $G$, the more localized they become.

\subsection{A local stability criterion}

When the degree $v$ is real, the solution (28) is radially non-oscillatory so that stability is ensured. This is the case when $D<1 / 4$, which yields for stability

$$
\left(1-\tilde{M}_{\mathrm{A}}^{2}\right) \frac{r}{4}\left(\frac{q^{\prime}}{q}\right)^{2}>-\beta^{\prime}\left(1-q^{2}\right)+q^{2}\left(S_{\gamma}+S_{k}+S_{\mathrm{f}}\right),
$$

where

$$
\begin{aligned}
& S_{\gamma}=G^{\prime} / m^{2}-\left(m^{2}-1\right) G / m^{2} r \\
& S_{k}=-\beta_{k}^{\prime}=-\left[\beta \mathcal{M}^{2}\left(1+\mathcal{M}^{2}\right)\right]^{\prime}, \\
& S_{\mathrm{f}}=2 \beta \mathcal{M}^{2}\left(1+\gamma^{-1} \mathcal{M}^{2}\right) \Omega^{2^{\prime}} / \Omega^{2} .
\end{aligned}
$$

Alternatively, the multiplicative factor $1-\tilde{M}_{\mathrm{A}}^{2}$ can be taken into account by adding $q^{2} S_{\mathrm{s}}$ to the right-hand side of (29), where

$$
S_{\mathrm{s}}=\frac{r}{4} \frac{\tilde{F}}{q^{2}} \frac{\Omega^{\prime 2}}{\omega_{\mathrm{A}}^{2}} .
$$

\subsection{Ordering}

Within the ordering used in the derivation of (4), the terms in the stability criterion (29) are all of order $\beta \sim \epsilon^{2}$. The dimensionless flow $\mathcal{M}$ and flow shear $\tilde{M}_{\mathrm{A}}$ are both of order unity. The conditions (22) used in the derivation of (29) introduce some additional restrictions on the quantities involved. The second inequality of (22) requires

$$
\left(1-\gamma^{-1}\right) \beta \mathcal{M}^{4} \ll\left|1-\tilde{M}_{\mathrm{A}}^{2}\right| r_{0}^{2}\left(\frac{n q^{\prime}}{q}\right)^{2} .
$$

Since $a^{2}\left(n q^{\prime} / q\right)^{2}$ is of the same order as $\beta$, the analysis breaks down when either $\left(r_{0} / a\right)^{2}$ or $\left|1-\tilde{M}_{\mathrm{A}}^{2}\right|$ becomes too small and (34) additionally requires

$$
1-\gamma^{-1} \ll 1 \quad \text { or } \quad \mathcal{M}^{4} \ll 1 .
$$

In the stability condition (29) $\gamma$ appears as a free parameter, whereas for high $\mathcal{M}$ (35) requires the use of $\gamma \approx 1$. We will look at the two options (35) explicitly in sections 5.2 and 5.3.

Note that for low $n$, assumption (34) makes $S_{\gamma}$ negligible in the stability criterion (29). For $n \gg 1$, the part $S_{\gamma} \approx-G / r$ may, however, still be relevant. This is because for $n \gg 1$, magnetic shear ensures mode localization even when $G$ increases.

When either $\mathcal{M}=0$ or $\gamma=1$, the continuum frequency $\omega_{-}$vanishes at the rational surface so that $\omega_{\mathrm{D}}=0$ becomes a cluster point. In these particular cases, the result (29) could have been obtained in a more formal fashion using the Frobenius method [45]. 
Using $S_{\gamma} \approx-G / r$ and $\beta \mathcal{M}^{2}=\Omega^{2} / \omega_{\mathrm{A}}^{2},(29)$ can be written as

$$
\begin{aligned}
\frac{r}{4}\left(\frac{q^{\prime}}{q}\right)^{2}>- & \beta^{\prime}+q^{2}\left[\beta\left(1-\mathcal{M}^{2}-\mathcal{M}^{4}\right)\right]^{\prime} \\
& +\frac{q^{2}}{\omega_{\mathrm{A}}^{2}}\left(r \tilde{F}\left(\frac{\Omega^{\prime}}{2 q}\right)^{2}+2 \Omega^{2^{\prime}}\left(1+\frac{\mathcal{M}^{2}}{\gamma}\right)-\left(1-\gamma^{-1}\right) \frac{\Omega^{2} \mathcal{M}^{2}}{r}\right) .
\end{aligned}
$$

\section{Physical interpretation}

In the absence of flow, stability criteria (29) or (36) reduce to a well-known expression for local stability, discussed in section 5.1. The left-hand side of (29) or (36) shows the stabilizing effect associated with the bending of magnetic field lines. The right-hand side contains the destabilizing pressure gradient $\beta^{\prime}$ and also a term $q^{2} \beta^{\prime}$ representing the average stabilizing effect of toroidal magnetic field curvature. The physical interpretation of the various flow terms will be discussed next.

\subsection{The convective term $S_{\gamma}$}

The assumption underlying the appearance of the stabilizing $S_{\gamma}$ term is that of adiabatic plasma motion conserving $S \equiv p \rho^{-\gamma}$. When the pressure adjusts instantaneously, a perturbed plasma element will have a relative density difference with its environment proportional to $-S^{\prime} / \gamma S$. A displacement in the direction of a force $g$ per unit mass will therefore yield an oscillation frequency $\omega_{\mathrm{BV}} \equiv \sqrt{-g S^{\prime} / \gamma S}$. This Brunt-Väisälä-frequency or buoyancy frequency describes stable oscillations when the specific entropy $S$ decreases in the direction of the body force. Otherwise its gives the growth rate of instability.

Plasma confined to an isothermal magnetic surface will encounter an increasing pressure (1) in the direction of the centrifugal force. When $\gamma>1$ the specific entropy will then be decreasing, having a stabilizing influence. The centrifugal force per unit mass $g=R \Omega^{2}$ so that, using (1), we can write

$$
\omega_{\mathrm{BV}}^{2}=-\frac{1}{2} \frac{R \Omega^{2}}{\gamma S}\left|\frac{\delta S}{\delta R}\right|_{\psi}=-\frac{1-\gamma}{\gamma} \frac{R \mathcal{M}^{2}}{R_{0}^{2}} R \Omega^{2} \approx\left(1-\gamma^{-1}\right) \mathcal{M}^{2} \Omega^{2},
$$

where an average directional factor $\left\langle\cos ^{2} \theta\right\rangle=1 / 2$ was included for plasma motion within the circular magnetic surfaces, because both the centrifugal force and the entropy gradients correspond to gradients in the $R$-direction. In terms of this frequency

$$
S_{\gamma} \approx-\frac{G}{r} \approx-\frac{1}{r} \frac{\omega_{\mathrm{BV}}^{2}}{\omega_{\mathrm{A}}^{2}}
$$

We note that at the rational surface, for $\mathcal{M} \ll 1$, we can write $\omega_{-}^{2} \approx \omega_{\mathrm{BV}}^{2} /\left(1+2 q^{2}\right)$, revealing the convective effect as the origin of this finite continuum frequency. The Pfirsch-Schlüter factor $1+2 q^{2}$ accounts for an increased effective inertia, due to the motion along the magnetic field that accompanies poloidal motion within the magnetic surfaces. The stabilizing centrifugal convective effect, or gyroscopic stabilization after [11], is considered for sawteeth in tokamaks in $[12-15]$.

\subsection{The kinetic energy term $S_{k}$}

Previously, this term was found in the study of ballooning modes [17], the internal kink mode [11] and localized modes [37,38]. The term corresponds to a variation of the kinetic 
energy density $(\partial / \partial r)\left(\boldsymbol{R} \cdot \nabla \frac{1}{2} \rho R^{2} \Omega^{2}\right) \approx\left[2 p_{\mathrm{s}} / \mathcal{M}^{2}\left(1+\mathcal{M}^{2}\right)\right]^{\prime}=-B_{0}^{2} S_{k}$, where (1) was used. Typically in a tokamak both $p_{\mathrm{s}}$ and $\mathcal{M}$ decrease radially, furthering instability by effectively diminishing the Mercier curvature term $q^{2} \beta^{\prime}$.

The origin of $S_{k}$ can be traced back to the last term in (3), which is associated with a potential energy $(\boldsymbol{\xi} \cdot \boldsymbol{R})^{*}(\boldsymbol{\xi} \cdot \nabla \psi) \partial \rho \Omega^{2} / \partial \psi[11]$. This shows that $S_{k}$ requires the perturbation to have a component in the direction of the centrifugal force.

The origin of a kinetic energy gradient as a source of instability can be understood by considering a thin differentially rotating disc with a small perpendicular magnetic field, e.g. an astrophysical accretion disc [46]. Adding the Brunt-Väisälä-frequency squared of an incompressible plasma perturbation $R \Omega^{2} \rho^{\prime} / \rho$ and the frequency squared $R \Omega^{2^{\prime}}$ of the magnetorotational instability $[47,48]$ yields $R\left(\rho \Omega^{2}\right)^{\prime} / \rho[49,50]$. Instability resulting from a gradient in the kinetic energy may therefore be understood as a combination of the magnetorotational instability and the convective instability.

The incompressible buoyancy-frequency $\rho^{\prime} R \Omega^{2} / \rho$ appears for the convective instability because it results from radial plasma perturbations, which are nearly incompressible due to the large energy associated with magnetic compression. This instability is analogous to the Rayleigh-Taylor instability of a fluid on top of a lighter fluid. In contrast to the magnetorotational instability, this instability is often observed in experiments, see, e.g. $[3,4,6,7]$.

\subsection{The flutter term $S_{\mathrm{f}}$}

Because this term derives from the radial variation of the Doppler shift $\omega_{\mathrm{D}}^{\prime}=n \Omega^{\prime}$ through $A^{\prime}$ it is expected to play a role only for non-axisymmetric perturbations. Typically in a tokamak $\Omega^{2^{\prime}}<0$ so that $S_{\mathrm{f}}$ is stabilizing.

A potential energy term proportional to $\rho \Omega^{2^{\prime}}$ was found in [17] and [11,51] in relation to ballooning modes and the internal kink mode, respectively.

In [17], an integrated form of $S_{\mathrm{f}}$ was associated with the kinetic energy of the fluttering motion occurring as the plasma flows past the perturbation. Its origin can be traced back to the Coriolis term in (3).

Different from $S_{\mathrm{s}}$ and the shear stabilization effect found for ballooning modes [18, 19], the sign of $\Omega^{\prime}$ is important for $S_{\mathrm{f}}$. In contrast to the magnetorotational instability, $S_{\mathrm{f}}$ is stabilizing for a radially decreasing angular frequency. It may therefore be very well possible to experimentally observe the influence of this term on stability.

\subsection{The shear term $S_{\mathrm{s}}$}

The prefactor $1-M_{\mathrm{A}}^{2}$ already appears in the cylindrical result $[34,35,52]$, with $F=1$. The quantity $M_{\mathrm{A}}$ is sometimes referred to as the Alfvén Mach number and gives the ratio between the flow shear and the frequency shear of Alfvén waves. When these shears match, an instability does not bend the field lines and the magnetic shear term vanishes. In this way, flow shear effectively diminishes the stabilizing influence of magnetic field line bending. This destabilizing effect of $S_{\mathrm{s}} \sim \rho \Omega^{\prime 2}$ is essentially that of the Kelvin-Helmholtz instability, modified by toroidicity, rotation and a magnetic field. For a torus, a similar term was found in [36,53] and in [37], where it was eventually neglected due to the used ordering. Experimentally this instability has been studied in magnetically confined plasmas mainly in $Q$-machines, see, e.g. [5-7].

The same quantity $1-M_{\mathrm{A}}^{2}$ with $F=1$ multiplies the highest derivative term in ballooning mode equations $[18,19]$ at marginal stability. For low flow shears this Kelvin-Helmholtz term 
was found to lead to shear destabilization [18, 21] of ballooning modes. For higher flow shears with $M_{\mathrm{A}} \approx 1$, however, complete stabilization was obtained due to the effect of flow shear on poloidal mode coupling [20]. Non-linearly, interchanges may also be stabilized by flow shear $[54,55]$.

Note that (20) shows a stabilizing influence of the convective effect on the KelvinHelmholtz instability for high $\mathcal{M}^{4}$. The stabilizing Brunt-Väisälä term $M_{\mathrm{B}}^{2}$, diminishes the destabilizing shear term $M_{\mathrm{A}}^{2}$ in such a way that the $\mathcal{M}^{4} / \gamma$ term in $F$ is replaced by $\mathcal{M}^{4} / \gamma^{2}$ in $\tilde{F}$.

\section{Various limiting cases}

\subsection{No flow: $\mathcal{M}=0$}

Without rotation, $S_{\gamma}=S_{k}=S_{\mathrm{f}}=0$ so that (29) reduces to the Shafranov-Yurchenko condition $[29,30]$

$$
\frac{r}{4}\left(\frac{q^{\prime}}{q}\right)^{2}>-\beta^{\prime}\left(1-q^{2}\right)
$$

which is a limiting case of the Mercier criterion [28] for large-aspect ratio plasmas with a circular cross-section. In the cylindrical limit $q \rightarrow 0$, the last term vanishes so that (39) reduces to Suydam's criterion [31] for static cylindrical plasmas.

\subsection{Some flow: $\mathcal{M}^{4} \ll 1$}

Neglecting terms proportional to $\mathcal{M}^{4}$, the stability criterion (29) can be written as

$$
\frac{r}{4}\left(\frac{q^{\prime}}{q}\right)^{2}>-\beta^{\prime}\left(1-q^{2}\right)+\frac{q^{2}}{\omega_{\mathrm{A}}^{2}}\left(\rho\left(\frac{\Omega^{2}}{\rho}\right)^{\prime}+\left(1+2 q^{2}\right) \frac{r \Omega^{\prime 2}}{4 q^{2}}\right),
$$

where $\rho\left(\Omega^{2} / \rho\right)^{\prime}=2 \Omega^{2^{\prime}}-\left(\rho \Omega^{2}\right)^{\prime} / \rho$ derives from the sum of $S_{\mathrm{f}}$ and $S_{k}$, respectively. When the angular frequency squared decreases faster than the density, this term is stabilizing. The shear term in (40), on the other hand, is always destabilizing. The net effect of rotation therefore depends crucially on the specific angular frequency and density profile.

Note that the condition $\mathcal{M}^{4} \ll 1$ is appropriate for most tokamak plasmas, where the rotation velocity is usually somewhat smaller than the ion sound velocity. The stability criterion (40) may be of some relevance to assess the influence of toroidal rotation on transport barriers, which typically have low magnetic shear but high flow shears. For high- $n$ ballooning modes, however, flow shear influences poloidal coupling, potentially leading to complete stabilization [18-20].

\subsection{Isothermal: $\gamma=1$}

When instabilities arise on a long enough timescale for heat exchange with their environment to take place, it may be appropriate to assume $\gamma=1$. In this case, $S_{\gamma}$ vanishes so that (29) gives with $\beta_{k}=\beta \mathcal{M}^{2}\left(1+\mathcal{M}^{2}\right)$

$$
\frac{r}{4}\left(1-M_{\mathrm{A}}^{2}\right)\left(\frac{q^{\prime}}{q}\right)^{2}>-\beta^{\prime}\left(1-q^{2}\right)+q^{2} \beta_{k}\left(\frac{2 \Omega^{2^{\prime}}}{\Omega^{2}}-\frac{\beta_{k}^{\prime}}{\beta_{k}}\right) .
$$

Apart from the new shear term involving $M_{\mathrm{A}}^{2}$, this stability criterion is equal to those of [37, 38] derived for $\gamma=1$. For $\mathcal{M}^{4} \ll 1, \beta_{k} \approx \beta \mathcal{M}^{2}=\rho \Omega^{2} / B_{0}^{2}=\Omega^{2} / \omega_{\mathrm{A}}^{2}$ so that (41) can be 
written as

$$
\frac{r}{4}\left(\frac{q^{\prime}}{q}\right)^{2}>-\beta^{\prime}+q^{2}\left(\beta-\beta_{k}\right)^{\prime}+\frac{2 q^{2} \Omega^{2^{\prime}}+r\left(1+2 q^{2}\right) \Omega^{\prime 2} / 4}{\omega_{\mathrm{A}}^{2}} .
$$

For a rigidly rotating plasma with $\gamma=1$ as well as $\mathcal{M}^{4} \ll 1$, the only effect of flow on localized modes is therefore the subtraction of the kinetic energy density from the static pressure in the toroidal curvature term.

\subsection{Cylindrical limit}

In the cylindrical limit $q \rightarrow 0$, the only remaining effect of flow is through the factor $1-M_{\mathrm{A}}^{2}$ multiplying the magnetic shear term, with $M_{\mathrm{A}}=\left(q / q^{\prime}\right) \Omega^{\prime} / \omega_{\mathrm{A}}$. The resulting stability criterion may be compared with the cylindrical result $[34,35,52]$ for purely longitudinal flow, where the same factor $1-M_{\mathrm{A}}^{2}$ appears.

The cylindrical result, however, contains an additional term with a trans-slow resonance for $M_{\mathrm{A}}^{2}=\gamma p /\left(B^{2}+\gamma p\right)$, which is absent in (29). This is due to the ordering assumed in the derivation of the mode equation (4). The missing term is $\mathrm{O}\left(B_{\theta}^{2} / B_{z}^{2}\right)=\epsilon^{2}$ smaller than the other terms, which is why it does not appear in the present large-aspect ratio result. This trans-slow resonance also disappears in a proper kinetic treatment [56]. Physically, within the present ordering there is no coupling between the $m, n$-Alfvén continuum modes and the slow-magnetosonic continuum, only with its $m \pm 1, n$-sidebands. This is due to the fact that in cylindrical geometry, the slow-magnetosonic sidebands cross at the rational surfaces, enhancing the interaction with the Alfvén continuum.

\section{Simulations}

To illustrate some aspects of the obtained stability criterion (29), we investigate the stability of a specific rotating equilibrium numerically. An analytical rigidly rotating isothermal equilibrium $[57,58]$ is used with $\gamma=5 / 3, \epsilon=0.1$, and $\beta$ between zero at the plasma edge and a maximum $\beta \approx 0.015$. This resulted in a monotonically increasing $q$-profile, centred around $q_{0}=1$ at $r_{0} \approx 0.6$, where $q^{\prime} \approx 0.023$. For this low shear equilibrium with constant $\Omega$ and $\mathcal{M}$, (29) predicts instability for $\mathcal{M} \ll 1$ when

$$
\mathcal{M}>\sqrt{-r q^{\prime 2} / 4 \beta^{\prime}} \approx 0.1
$$

The ideal MHD spectrum of waves and instabilities of this equilibrium was investigated with PHOENIX [59], including seven poloidal harmonics in the eigenvalue calculation. Convergence checks with more poloidal harmonics have been performed to ensure the accuracy of the results.

Local stability criteria are sufficient conditions for stability only for modes that are localized radially. In particular for high mode numbers, modes will also localize poloidally at the low-field side. We may therefore expect (29) to be a better guide for stability at low mode numbers, which is what we will focus on. As discussed at the end of section 3.2, mode localization in this case has to come primarily from closeness of the mode frequencies to the continuum.

\subsection{Quasi-interchange mode}

For $m=n=1$, a Sturmian sequence of unstable modes appears above a Mach number $M_{0}=\sqrt{5 / 6} \mathcal{M} \approx 0.15$, consistent with (43). Figure $1(b)$ shows the growth rate of these 

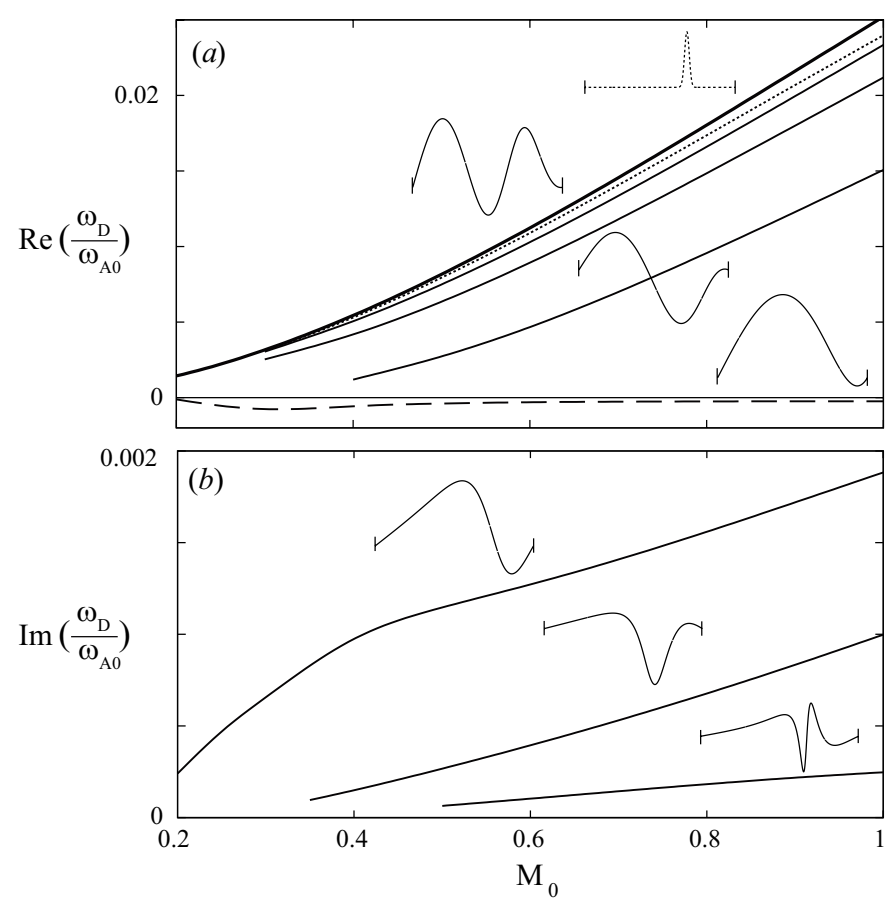

Figure 1. (a) The Doppler-shifted frequency $\omega_{\mathrm{D}}$ divided by $\omega_{A 0} \equiv \omega_{\mathrm{A}}\left(R=R_{0}\right)$, of the $\omega_{-}$-continuum (thick line), the first zonal flow mode (dots), the first three global Alfvén modes (solid), and the most unstable interchange mode (dashes). (b) The dimensionless growth rate of the most unstable modes as a function of $M_{0}=\sqrt{5 / 6} \mathcal{M}$. The radial perturbation of the stable and unstable modes and the poloidal perturbation of the zonal flow-modes is displayed for $M_{0}=1$. Note that the smaller the growth rate of the unstable modes, the more localized they are.

unstable quasi-interchange modes for various Mach numbers $M_{0}$. The real part of the Dopplershifted frequency of the instability, the Coriolis shift [44], is not monotonic in $M_{0}$, as shown in figure 1(a). It does become very small for marginally stable modes, justifying the use of $\omega_{\mathrm{D}}=0$ in the performed stability analysis.

\subsection{Other modes}

Figure 1(a) also shows two types of modes reported earlier [58]. A Sturmian sequence of modes is present, which have a finite amplitude only in a small range around the rational surface and are polarized within the magnetic surfaces. The frequency and characteristics of these modes allowed us to identify them as a type of non-axisymmetric zonal flow modes [58]. The frequency of these modes is always somewhat below the continuum frequency $\omega_{\mathrm{D}}^{2}=\omega_{-}^{2}$, so that these modes were found to become slightly unstable for low $M_{0}$.

The stable global rotation-induced Alfvén modes discussed in [58] appear above $M_{0} \approx 0.15$, along with the unstable modes. These modes also cluster below the $\omega_{-}$-continuum frequency as shown in figure $1(a)$. The mode structure of the unstable modes is very similar to that of these stable Alfvén modes. Both modes have a single dominant poloidal mode number that is resonant with the rational surface and small sideband amplitudes.

The most significant difference is in the parallel component, which is much larger for the unstable modes. The unstable modes apparently couple more strongly with slow-magnetosonic 


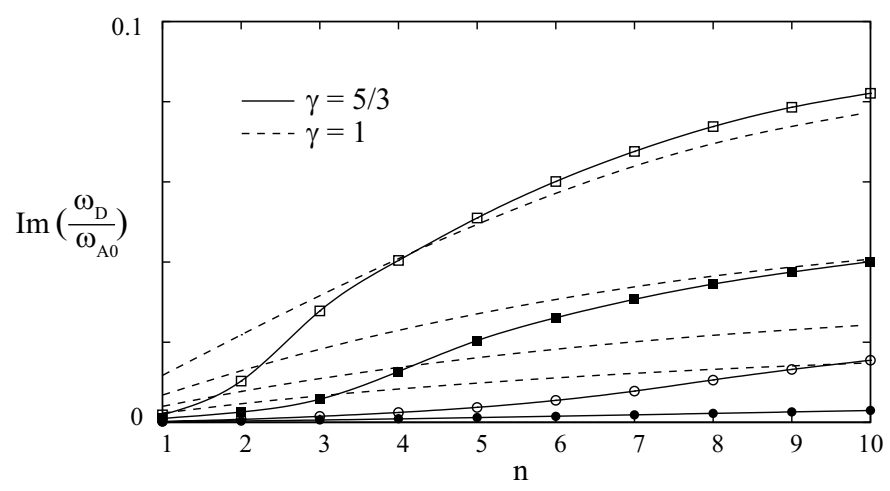

Figure 2. The growth rate of the first few unstable modes as a function of $n=m$ for $M_{0}=1$. Here $\omega_{A 0}$ is $\omega_{\mathrm{A}}$ evaluated at the magnetic axis.

waves to form a mixed slow-Alfvén eigenmode. For higher mode numbers, the radial component becomes more significant compared with the poloidal component. Also the sideband amplitudes of the parallel component become more and more significant for higher mode numbers. For $n=10$ e.g., the $m=9$ and $m=11$ side bands of the parallel displacement become an order of magnitude larger than the main $m=10$ component.

\subsection{Infernal modes}

Figure 2 shows, for $M_{0}=1$, the growth rate of the first few most unstable modes as the toroidal mode number $n$ is increased from 1 to 10 . The growth rate increases by almost two orders of magnitude, to reach a dangerously high growth rate of $\operatorname{almost} \operatorname{Im}\left(\omega_{\mathrm{D}}\right) \approx 0.1 \omega_{\mathrm{A}}$ for $n=10$.

With increasing $n$, coupling to poloidal sideband harmonics becomes more important. For the present case, even for $n=10$, the neighbouring rational surfaces at $q=(n \pm 1) / n$ lie outside the plasma due to the low magnetic shear. The mode can minimize magnetic field line bending by shifting its poloidal sidebands in the direction of their respective rational surface. This effect is indeed observed in the simulations. These sideband amplitudes have opposite sign, to give localization at the low-field side. This weak ballooning is visible primarily in the parallel displacement, the component that does not bend the field lines.

In figure 2 the growth rate is shown for the same equilibrium, using $\gamma=1$ in the stability calculations. The effect of $\gamma$ is seen to be largest for modes with a higher number of nodes. The stabilization is also most effective for low- $m, n$ modes, which may be explained by the term $S_{\gamma}$ in (30). The modes shown in figure 2 are far from marginality and not localized enough to justify the analysis of section 3. The Mercier index $D$ from (26) may, however, be used as a first approximation for the terms multiplying $\xi$ in the mode equation (4). The term (30) contains a part inversely proportional to $m$ that is stabilizing when $G^{\prime}<-G / r$. For the present equilibrium this requires $\left|\beta^{\prime}\right|>\beta / r$ which, for $r \approx r_{\mathrm{s}} \approx 0.6$ is indeed the case.

Another observation from the data in figure 2 is that the growth rate decreases approximately exponentially with the number of nodes of the unstable modes, in agreement with the result from a boundary layer analysis [60]. Finally we note that for $n=10$, unstable modes are already present in the absence of flow, in contrast with (39). This again shows that local criteria should not be interpreted as sufficient conditions for stability, since they do not properly take into account mode coupling. 


\section{Conclusions and discussion}

A local stability criterion (29) has been derived including the effects of toroidal rotation and rotation shear. This stability criterion reduces to various known limits. A trans-slow resonance is absent, because in the assumed ordering the Alfvén waves only couple to the slowmagnetosonic sidebands. A general adiabatic index $\gamma$ is retained in the analysis, although for sonic rotation velocities the analysis allows only small deviations from isothermality. For $\gamma>1$, the accumulation point for localized modes increases in frequency through the centrifugal convective effect. Destabilizing rotational effects may, however, drive the most unstable modes away in frequency faster from the accumulation point than this frequency moves away from marginality. A destabilizing effect is e.g. associated with flow shear. A further destabilizing term is present when the density decreases faster than the angular frequency squared.

The derived criterion is only a sufficient condition for stability for modes that are localized radially but not poloidally. Local criteria of this kind may therefore be regarded as necessary conditions for stability only. Numerical simulations indeed show this to be the case. Poloidal harmonics couple to create an additional destabilizing outward ballooning effect. In relation to this point, we note the work of [61] showing that for a static cylindrical plasma within ideal MHD the effect of magnetic shear is to localize instabilities rather than to stabilize them. This is at variance with local criteria like those of Suydam and that derived in this work, showing once more the caution that should be taken in interpreting local stability criteria.

The instabilities considered in this paper are particularly unstable in regions of low magnetic shear, existing e.g. in 'hybrid' advanced tokamak scenarios. These instabilities can also arise due to the very high pressure gradients near internal transport barriers, which typically arise near low mode number rational surfaces and involve low magnetic shear and high flow shear [18]. The work of $[62,63]$ shows that a local stability criterion is equal to the existence criterion for reversed shear Alfvén eigenmodes near a rational surface. The modifications due to flow discussed here, may therefore also be relevant for these modes that appear as Alfvén cascades and are used for MHD spectroscopy [64].

\section{Acknowledgments}

The authors thank J P Goedbloed for his constructive criticism and continued interest. This work, supported by NWO and the European Communities under the contract of the Association EURATOM/FOM, was carried out within the framework of the European Fusion Programme. The views and opinions expressed herein do not necessarily reflect those of the European Commission.

Euratom (C) 2011.

\section{References}

[1] Chu M S and Okabayashi M 2010 Plasma Phys. Control. Fusion 52123001

[2] La Haye R J et al 2010 Phys. Plasmas 17056110

[3] Tuszewski M et al 1990 Phys. Fluids B 22541

[4] Levitt B, Maslovsky D, Mauel M E and Waksman J 2005 Phys. Plasmas 12055703

[5] D'Angelo N and Goeler S V 1966 Phys. Fluids 9309

[6] Jassby D L 1972 Phys. Fluids $\mathbf{1 5} 1590$

[7] Sugai H et al 1977 Phys. Fluids 2090

[8] Garbet X et al 1999 Phys. Plasmas 63955

[9] Schwander F et al 2010 J. Nucl. Mater. doi:10.1016/j.jnucmat.2010.10.073 submitted

[10] Sugita S et al 2010 J. Phys. Soc. Japan 79044502 
[11] Waelbroeck F L 1996 Phys. Plasmas 31047

[12] Chapman I T et al 2006 Nucl. Fusion 461009

[13] Chapman I T et al 2007 Plasma. Phys. Control. Fusion 49 B358 doi:10.1088/0741-3335/49/12B/S35

[14] Chapman I T et al 2008 Nucl. Fusion 48035004

[15] Chapman I T et al 2010 Nucl. Fusion 50045007

[16] Hameiri E and Laurence P 1984 J. Math. Phys. 25396

[17] Waelbroeck F L and Chen L 1991 Phys. Fluids B 3601

[18] Webster A J and Wilson H R 2004 Phys. Rev. Lett. 92165004

[19] Miller R L, Waelbroeck F L, Hassam A B and Waltz R E 1995 Phys. Plasmas 23676

[20] Furukawa M and Tokuda S 2005 Phys. Rev. Lett. 94175001

[21] Connor J W, Hastie R J and Webster A J 2007 Phys. Plasmas 14044504

[22] Manickam J, Pomphrey N and Todd A M M 1987 Nucl. Fusion 271461

[23] Ozeki T, Azumi M, Tokuda S and Ishida S 1993 Nucl. Fusion 331025

[24] Zakharov L E 1978 Nucl. Fusion 18335

[25] Charlton L A, Hastie R J and Hender T C 1989 Phys. Fluids B 1798

[26] Wesson J A 1986 Plasma. Phys. Control. Fusion 28243

[27] Waelbroeck F L and Hazeltine R D 1988 Phys. Fluids 311217

[28] Mercier C 1960 Nucl. Fusion 147

[29] Ware A A and Haas F A 1966 Phys. Fluids 9956

[30] Shafranov V D and Yurchenko E I 1968 Sov. Phys._JETP 26682

[31] Suydam B R 1959 Proc. 2nd UN Int. Conf. on Peaceful Uses of Atomic Energy 31 (New York: Columbia University Press) p 157

[32] Goedbloed J P 1973 Phys. Fluids 161927

[33] Gupta S, Callen J D and Hegna C C 2002 Phys. Plasmas 93395

[34] Hameiri E 1981 J. Math. Phys. 222080

[35] Bondeson A, Iacono R and Bhattacharjee A 1987 Phys. Fluids 302167

[36] Chu M S 1998 Phys. Plasmas 5183

[37] Zheng L J, Chu M S and Chen L 1999 Phys. Plasmas 61217

[38] Wahlberg C and Bondeson A 2001 Phys. Plasmas 83595

[39] Wahlberg C and Bondeson A 2000 Phys. Lett. A 271285

[40] Wahlberg C 2005 Plasma Phys. Control. Fusion 47757

[41] Wahlberg C 2009 Plasma Phys. Control. Fusion 51085006

[42] Frieman E and Rotenberg M 1960 Rev. Mod. Phys. 32898

[43] van der Holst B, Beliën A J C and Goedbloed J P 2000 Phys. Plasmas 74208

[44] Goedbloed J P 2009 Phys. Plasmas 16122110

[45] Bender C M and Orszag S A 1978 Advanced Mathematical Methods for Scientists and Engineers (New York: McGraw-Hill)

[46] Blokland J W S, van der Swaluw E, Keppens R and Goedbloed J P 2005 Astron. Astrophys. 444337

[47] Velikhov E P 1959 Sov. Phys._JETP 36995

[48] Balbus S A and Hawley J F 1991 Astrophys. J. 376214

[49] Sen A K 1994 Nucl. Fusion 34459

[50] Furukawa M, Yoshida Z, Hirota M and Krishan V 2007 Plasma Fusion Res. 2016

[51] Wahlberg C, Chapman I T and Graves J P 2009 Phys. Plasmas 16112512

[52] Hameiri E 1976 The Stability of a Particular MHD Equilibrium with Flow PhD Thesis New York University

[53] Hegna C C 2003 Linear resistive layer equations in the presence of sheared toroidal rotation Technical Report UW-CPTC 03-5

[54] Hassam A B 1992 Phys. Fluids B 4485

[55] Huang Y M and Hassam A B 2001 Phys. Rev. Lett. 87235002

[56] Bondeson A and Iacono R 1989 Phys. Fluids B 11431

[57] Maschke E K and Perrin H 1980 Plasma Phys. 22579

[58] Haverkort J W, de Blank H J and Koren B 2011 Plasma Phys. Control. Fusion submitted

[59] Blokland J W S, van der Holst B, Keppens R and Goedbloed J P 2007 J. Comput. Phys. 226509

[60] Grad H 1973 Proc. Natl Acad. Sci. USA 703277

[61] Longaretti P-Y 2003 Phys. Lett. A 320215

[62] Breizman B N and Pekker M S 2005 Phys. Plasmas 12112506

[63] Fu G Y and Berk H L 2006 Phys. Plasmas 13052502

[64] Goedbloed J P, Holties H A, Poedts S, Huysmans G T A and Kerner W 1993 Plasma Phys. Control. Fusion 35 B277 